\title{
Global circumferential left ventricular strain impairment in hypertrophic cardiomyopathy: comparison to left ventricular hypertrophy and late gadolinium enhancement
}

\author{
Laurent Macron ${ }^{1 *}$, Alban Redheuil $^{1,3}$, Golmehr Ashrafpoor ${ }^{1,2}$, Nadjia Kachenoura ${ }^{3}$, Emilie Bollache ${ }^{3}$,
} Albert A Hagège ${ }^{2}$, Michel Desnos², Pierre Croisille ${ }^{4}$, Patrick Clarysse ${ }^{4}$, Elie Mousseaux ${ }^{1,3}$

From 16th Annual SCMR Scientific Sessions

San Francisco, CA, USA. 31 January - 3 February 2013

\section{Background}

to evaluate the relationship between Left Ventricular (LV) myocardial strain, mass, wall thickness and the extent of fibrosis in hypertrophic cardiomyopathy $(\mathrm{HCM})$

\section{Methods}

Forty HCM patients and 20 matched controls underwent a comprehensive CMR including cine imaging, late gadolinium enhancement (LGE) and short axis tagging. Global peak circumferential LV strain (Ecc) was generated from tagging sequences using InTag ${ }^{\circledR}$. LGE volume was quantified semi automatically using a 6SD threshold.

\section{Results}

HCM patients $(50 \pm 18$ years, $65 \%$ men) had normal LVEDV $(149 \pm 46 \mathrm{~mL}, \mathrm{p}=0.24)$, LVESV $(52 \pm 24 \mathrm{~mL}, \mathrm{p}=0.78)$ and LVEF $(65 \pm 11 \%, \mathrm{p}=0.38)$. LV mass $(198 \pm 69 \mathrm{~g}, \mathrm{p}<0.001)$ and LV mass index $(108 \pm 37 \mathrm{~g} / \mathrm{m} 2 \mathrm{p}=0.002)$ were significantly increased, resulting in decreased LV mass/LV volume ratio $(1.40 \pm 0.54, \mathrm{p}=0.005)$ in $\mathrm{HCM}$ compared to controls. Median maximal wall thickness was 19.6 (14.4 to $32.3 \mathrm{~mm})$. In HCM, LGE was present in 32/40 (80\%) and mean LGE mass was $4.31 \pm 4.94 \mathrm{~g}$.

Ecc was significantly impaired in HCM patients $(-8.82$ \pm 0.32 vs. $-15.54 \pm 2.54 \%, \mathrm{p}<0.0001$ )

Ecc impairment was significantly associated with increased LV mass index $(\mathrm{r}=0.51, \mathrm{p}=0.0009), \mathrm{LV}$ mass/ $\mathrm{LV}$ volume ratio $(\mathrm{r}=0.67, \mathrm{p}<0.0001)$ and $\mathrm{LV}$ maximal wall thickness $(\mathrm{r}=0.51, \mathrm{p}=0.008)$. Moreover, Ecc impairment was associated with increased LGE mass $(r=0.39, p=0.01)$.

\section{Conclusions}

Global LV circumferential myocardial deformation was strongly decreased in HCM and significantly associated with LV hypertrophy and the extent of LGE.

\section{Funding}

none

\section{Author details}

'Radiology, Cardiovascular Imaging Unit, HEGP,APHP, Paris, France. ${ }^{2}$ Cardiology, HEGP,APHP, Paris, France. ' ${ }^{3}$ LIF, INSERM U678, UPMC, Paris, France. ${ }^{4}$ CREATIS, UMR CNRS 5220 - INSERM U1044, Lyon, France.

Published: 30 January 2013

doi:10.1186/1532-429X-15-S1-E122

Cite this article as: Macron et al.: Global circumferential left ventricular strain impairment in hypertrophic cardiomyopathy: comparison to left ventricular hypertrophy and late gadolinium enhancement. Journal of Cardiovascular Magnetic Resonance 2013 15(Suppl 1):E122.

${ }^{1}$ Radiology, Cardiovascular Imaging Unit, HEGP,APHP, Paris, France Full list of author information is available at the end of the article

(C) 2013 Macron et al; licensee BioMed Central Ltd. This is an Open Access article distributed under the terms of the Creative Commons Attribution License (http://creativecommons.org/licenses/by/2.0), which permits unrestricted use, distribution, and reproduction in any medium, provided the original work is properly cited. 